\title{
Lips Recognition for Biometrics
}

\author{
Michał Choraś \\ Institute of Telecommunications \\ University of Technology \& Life Sciences, Bydgoszcz, Poland \\ chorasmQutp.edu.pl
}

\begin{abstract}
One of the most interesting emerging method of human identification, which originates from the criminal and forensic practice, is human lips recognition. In this paper we consider lips shape features in order to determine human identity. The major contribution of this paper are novel geometrical parameters developed in order to describe human lips shape for biometric applications.
\end{abstract}

\section{Rationale}

Biometric methods of human identification have gained much attention recently, mainly because they easily deal with most problems of traditional identification. In biometric human identification systems users are identified by who they are, not by something they have to remember or carry with them.

Nowadays, there are many well known, already implemented methods of human identification (face, iris, retina, etc.), but still novel and innovative solutions are proposed and needed. Such situation is most apparent due to a large-scale biometrics problem, where even very successful methods fail in real-life security systems, such as access control in airports.

Even strong methods with errors of $F N M R=2 \%$ cause problems when deployed in a large scale: for 100000 passengers a day, such error effects in 2000 false rejects per day!

The problem is that 99,99\% accuracy in laboratory conditions does not result in the same accuracy and efficiency in a large scale and non-controlled, real-life environments. So far the scaling issues in biometrics are not formalized or solved.

Therefore, to cover up for possible errors, other biometric modalities should be used, so that the overall accuracy and reliability of the hybrid systems is higher. Even though, these new emerging methods do not give results comparable with face or fingerprint systems, still such methods may improve accuracy of the hybrid systems, especially for people for whom standard systems fail to be effective.

Current trends in searching for novel modalities to identify humans are:

- 3D imaging (face, finger, ear),

- thermal imaging (face) and

- forensic science.

M. Tistarelli and M.S. Nixon (Eds.): ICB 2009, LNCS 5558, pp. $1260-1269,2009$.

(C) Springer-Verlag Berlin Heidelberg 2009 
Some of the methods recently termed as "emerging" have now matured, to mention ear biometrics (motivated by forensic science).

Other new and emerging biometric modalities are [1] [2] [4]:

- human scent recognition,

- EEG biometrics,

- skin spectroscopy,

- knuckles texture,

- finger-veins and

- finger-nails recognition.

The major contribution of this paper are novel geometrical parameters developed in order to describe human lips shape for biometrics.

In our approach we calculate color features and we merge them with shape features of the binarized lips. We calculate color statistics and moments as well as a set of standard geometrical parameters and the moments of $\mathrm{Hu}$ and Zernike. Moreover, we developed original lips shape descriptors that enhance the effectiveness of our lips recognition method for biometric applications.

Lips recognition as biometrics is introduced and motivated in Section 2, In Section 3 related research developments are briefly overviewed. In Section 4.1 our algorithm for lips detection with sample results is presented. Original shape features developed for lips biometrics are proposed in Section 4.3. Experimental results, future work and conclusions are given thereafter.

\section{Lips Biometrics}

One of the most interesting emerging method of human identification, which originates from the criminal and forensic practice (cheiloscopy), is human lips recognition [5] 6].

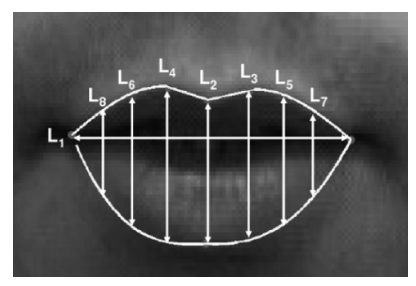

Fig. 1. Lips shape parameters proposed by Cetingul et al. [1]

The fact that lips features may be unique for humans have been confirmed by Yasuo Tsuchihasi and Kazuo Suzuki in their studies at Tokio University (19681971). They examined 1364 subjects from 3 to 60 years of age of both genders. Their research proved that lips characteristics are unique and unchangeable (stable) for each examined person [5] 7 . 
In another research lip-prints have been used to support the sex determination of the examined subject [8].

Lip-prints characteristics have been also used in forensics experts and criminal police practice. Such approach to human identity confirmation is used by the police and have been included as a subdiscipline of dactyloscopy.

Human lips features may be implemented in various scenarios, such as:

- speech recognition,

- multimodal audio-video speech recognition,

- speaker identification,

- multimodal audio-video speaker identification,

- lip-reading and finally,

- lips based human identification based on the static mouth/face images.

In general, in accordance to the application scenario, lips features can be divided into three different categories: lips texture features, lips shape features and lips motion features.

Hereby we consider human identification based on the static mouth/face images.

Using lips as modality for human identification has the following advantages:

1. Lips biometrics is passive biometrics - user/subject interaction is not needed. Images may be acquired from the distance without the knowledge of the examined person.

2. Lips biometrics is anatomical - better results are expected than in behavioral biometrics.

3. Lips are usually visible - not hidden/overcast by anything.

4. Lips may be implemented in hybrid lips-face or lips-voice biometric systems.
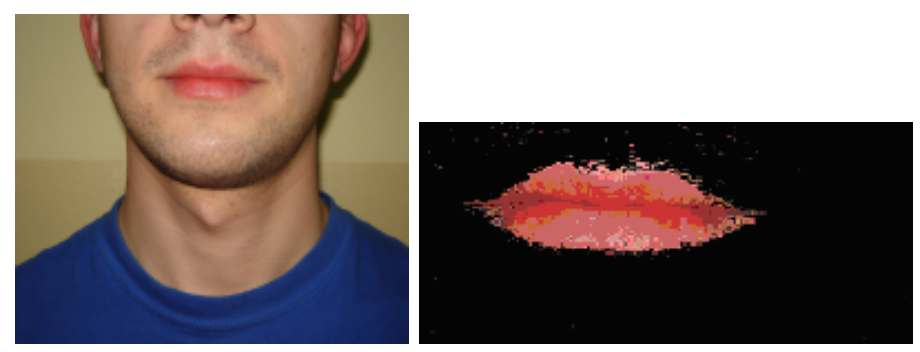

Fig. 2. Example of lower face and extracted corresponding lips area

\section{Related Work}

While manually examining human lips characteristics, most often, the anatomical patterns on the lips are taken into account. For example the pioneer of cheiloscopy, professor J. Kasprzak defined 23 lip patterns [5]. Such patterns 
(lines, bifurcations, bridges, pentagons, dots, lakes, crossings, triangles etc.) are similar to fingerprint, iris or palmprint patterns.

However, in biometric systems based on image analysis techniques, such linetopology features cannot be used since it is hard to extract them from acquired images.

Therefore in our approach we do not use lip-prints features, but we focus on characteristics extracted from lips/face images. Such images can be acquired from a certain distance by a standard camera in controlled or uncontrolled environment.

Automated lips biometrics have not been extensively researched so far, but there are some published approaches to human lips feature extraction.

Gomez et al. achieved some very promising results using geometrical parameters, $H M M$ and $P C A$ methods [9]. They reported $96.2 \%$ recognition rate on a database of 500 images (50 subjects with 10 images per person).

Cetingul et al. proposed to explore $2 d-D C T$ coefficients as lips texture features and used them in multimodal speaker/speech recognition system [10].

They also developed eight lips shape parameters based on the lips geometry. The maximum horizontal distance and the 7 vertical distances (from the Cupid's bow to the lower lip boundary characteristic points) are calculated (Figure 1) 11].

\section{A Geometrical Approach to Lips Biometrics}

\subsection{Lips Detection}

In the first step lips are detected from face images. Then we perform segmentation, binarization and size normalization. Most of known lips detection methods proposed so far, had been designed for speech recognition, lips tracking and lip-reading 11 12,13 14 15 16.

We modified a method based on color discriminates proposed by Kubanek [17. and we applied it to the $H S V$ color space.

The condition for lips detection is presented in Eq1.

$$
\left\{\begin{array}{l}
H \geq 334 \vee H \leq 10 \\
S \leq 25
\end{array}\right.
$$

The sample results of our lips detection algorithm from lower face images are presented in Figure 2.

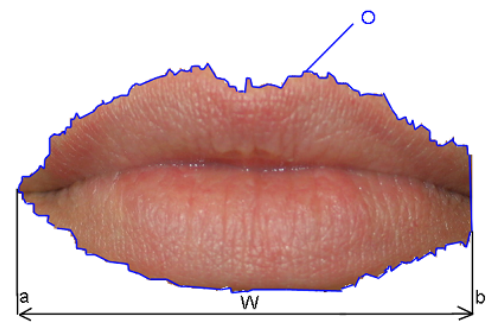

Fig. 3. Lips width to perimeter ratio 


\subsection{Lips Shape Feature Extraction}

After lips detection stage, shape features of the binarized lips images are calculated.

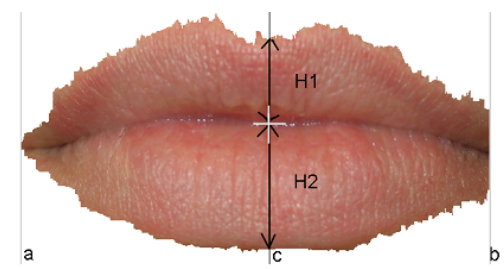

Fig. 4. Upper to lower lip height ratio

We decided to calculate geometrical parameters of detected lips area. In our previous work, we calculated central moments, Zernike moments and Hu moments [18. Moreover, we used standard geometrical shape parameters to describe human lips [19:

- Malinowska ratio

- Feret ratio

- Blair-Bliss ratio

- Danielsson ratio

- Haralick ratio

- Lp1 ratio

- Lp2 ratio.

\subsection{Original Lip Shape Parameters}

Furthermore, in this paper we present our own original parameters developed for the lips biometrics.

9 novel parameters, developed especially for lips biometrics and describing lips' shape are as follows:

1. lips width to perimeter ratio $W O$ given by: $W O=W / O$ where $W$ is the lips width and $O$ is lips perimeter, as presented in Figure 3

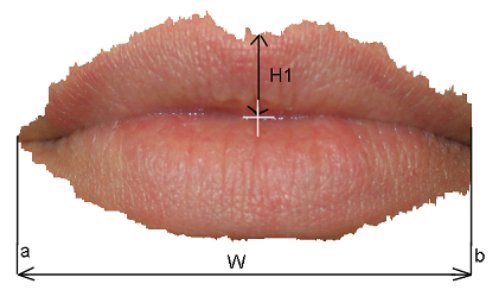

Fig. 5. Upper lip height to width ratio 


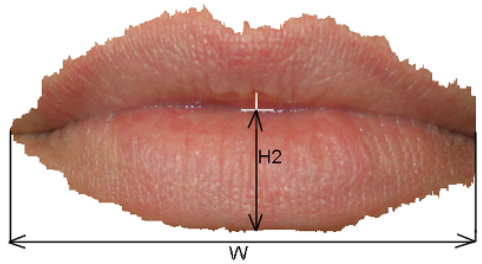

Fig. 6. Lower lip height to width ratio

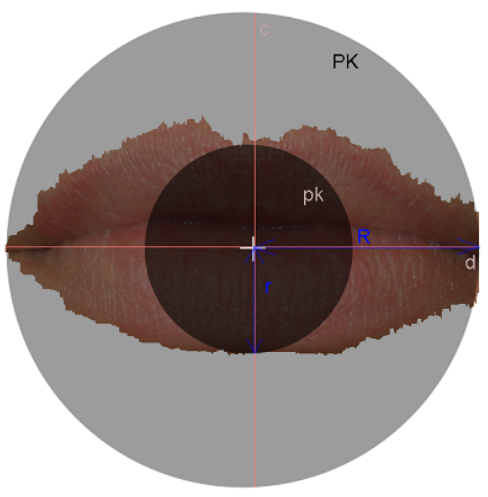

Fig. 7. Inner to outer circle ratio

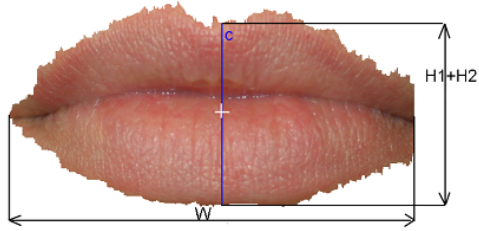

Fig. 8. Width to middle height ratio

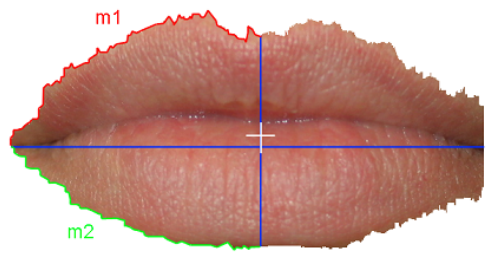

Fig. 9. Left side upper to lower lip convexity ratio 


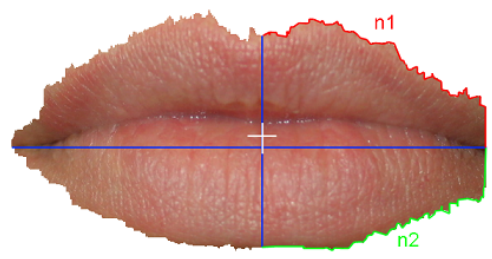

Fig. 10. Right side upper to lower lip convexity ratio

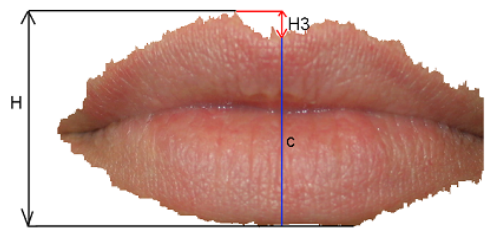

Fig. 11. Indent ratio

2. Upper to lower lip height ratio $U L H$ given by: $U L H=H 1 / H 2$ where $H 1$ is the upper lip height calculated in the middle column of the lips, and $H 2$ is the lower lip height calculated in the middle column of the lips (Figure 4).

3. Upper lip height to width ratio $U L W$ given by: $U L W=H 1 / W$ where $H 1$ is the upper lip height calculated in the middle column of the lips and $W$ is the lips width (Figure 5).

4. Lower lip height to width ratio $L L W$ given by: $L L W=H 2 / W$ where $H 2$ is the upper lip height calculated in the middle column of the lips and $W$ is the lips width (Figure 6).

5. Inner to outer circle ratio $C 2 C$ given by: $C 2 C=p k / P K$ where $p k$ is the inner circle and $P K$ is the outer circle as shown in Figure 7.

6. Width to middle height ratio $W M H$ given by: $W M H=W /(H 1+H 2)$ as presented in Figure 8 .

7. Left side upper to lower lip convexity ratio $L C$ given by: $L C=m 1 / m 2$ where $m 1$ is the upper lip left side convexity and $m 2$ is lower lip left side convexity as presented in Figure 9 .

8. Right side upper to lower lip convexity ratio $R C$ given by: $R C=n 1 / n 2$ where $n 1$ is the upper lip left side convexity and $n 2$ is lower lip left side convexity as presented in Figure 10.

9. Indent ratio $I R$ given by: $I R=H 3 / H$ where $H$ is the lips height and $H 3$ is the height of the indent calculated according to the Figure 11.

\section{Experimental Results}

In the experiments, we used our own database of lower face images. We acquired 3 images per person. The lips database used in our experiments consists of 3 images from 38 people (114 images). 
After experiments and evaluation it turned out that adding novel geometrical parameters to the feature vector increased system's efficiency (in comparison to our previous work (without novel geometrical parameters) [18] 19]).
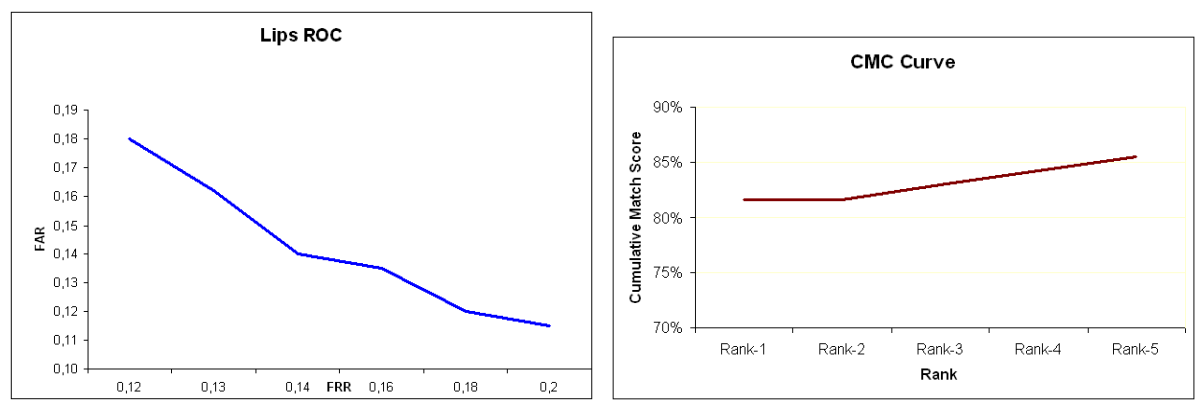

Fig. 12. ROC-Curve (left) and CMC-curve (right) of lips biometric system

Human lips recognition results without and with the novel developed geometrical parameters are shown in the Table 1.

Table 1. Lips Rank-1 recognition rate results

\begin{tabular}{|c||c|c|c||c|}
\hline method & number of tests & correct acceptances & false rejections & Rank-1 \\
\hline without novel parameters & 76 & 58 & 18 & $76 \%$ \\
\hline with novel parameters & 76 & 62 & 14 & $82 \%$ \\
\hline
\end{tabular}

So far we have achieved the recognition rate (Rank-1) of $82 \%$.

The ROC Curve of our lips biometrics system is presented in Figure 12 -left.

The CMC curve describing our system is presented in Figure 12 right. The Rank-4 and Rank-5 recognition rate increased up to $86 \%$.

At this moment we work on using symmetry as a feature which may help to better describe human lips.

We use horizontal and vertical symmetry to obtain 2 symmetrical images and 2 'delta' images (difference between the originally acquired subject's image and corresponding symmetry images).

Now we work on selecting efficient features from symmetry and delta images. We also plan to use Discrete Symmetry Transform to find lips fiducial points to which Gabor Jets may be applied [20].

\section{Conclusions}

In this paper we presented feature extraction methods and introductory results of automated human lips biometric system.

In our research novel lips shape descriptors had been used to determine human identity. 
We have achieved promising recognition results for well detected lips images, which motivate our further research in this area.

We plan to extend our research with the development of new lips features. We are investigating the texture and symmetry features of the human lips. Moreover, we try to improve the lips detection algorithm with the Active Shape Models approach.

Lips biometrics could be used to enhance effectiveness of other well-known biometrics, by its implementation in multimodal systems. Since most of the methods have some drawbacks, the idea of building multimodal (hybrid) biometric systems is gaining lot of attention [21].

Lips biometrics seems to be a natural choice to support well known methods like voice and face recognition in emerging applications such as access control, border security and recognition for human-vehicle interaction [22].

\section{References}

1. Prabhakar, S., Kittler, J., Maltoni, D., O'Gorman, L., Tan, T.: Introduction to the Special Issue on Biometrics: Progress and Directions. IEEE Trans. on PAMI 29(4), 513-516 (2007)

2. Goudelis, G., Tefas, A., Pitas, I.: On Emerging Biometric Technologies. In: Proc. of COST 275 Biometrics on the Internet, Hatfield, UK, pp. 71-74 (2005)

3. Morales, A., Ferrer, M.A., Travieso, C.M., Alonso, J.B.: A knuckles texture verification method in a transformed domain. In: Proc. of 1st Spanish Workshop on Biometrics, Girona, Spain (2007) (on CD)

4. Choraś, M.: Emerging Methods of Biometrics Human Identification. In: Proc. of ICICIC 2007 - Kummamoto, Japan. IEEE CS Press, Los Alamitos (2007)

5. Kasprzak, J., Leczynska, B.: Cheiloscopy. Human Identification on the Basis of Lip Prints (in Polish). CLK KGP Press, Warsaw (2001)

6. Kasprzak, J.: Forensic Otoscopy (in Polish). University of Warmia and Mazury Press (2003)

7. Tsuchihasi, Y.: Studies on Personal Identification by Means of Lip Prints. Forensic Science 3(3) (1974)

8. Sonal, V., Nayak, C.D., Pagare, S.S.: Study of Lip-Prints as Aid for Sex Determination. Medico-Legal Update 5(3) (2005)

9. Gomez, E., Travieso, C.M., Briceno, J.C., Ferrer, M.A.: Biometric Identification System by Lip Shape. In: Proc. of Carnahan Conference on Security Technology, pp. 39-42 (2002)

10. Cetingul, H.E., Yemez, Y., Erzin, E., Tekalp, A.M.: Multimodal speaker/speech recognition using lip motion, lip texture and audio. Signal Processing 86, 35493558 (2006)

11. Cetingul, H.E., Yemez, Y., Erzin, E., Tekalp, A.M.: Discriminative Analysis of Lip Motion Features for Speaker Identification and Speech-Reading. IEEE Trans. Image Processing 15(10), 2879-2891 (2006)

12. Ouyang, H., Lee, T.: A New Lip Feature Representation Method for Video-based Bimodal Authentication. In: Proc. of NICTA-HCSNet Multimodal User Interaction Workshop, Sydney, Australia, vol. 57, pp. 33-37 (2005)

13. Leung, S.H., Wang, S.L., Lau, W.H.: Lip Image Segmentation Using Fuzzy Clustering Incorporating an Elliptic Shape Function. IEEE Trans. Image Processing 13(1), 51-62 (2004) 
14. Nowak, H.: Lip-reading with Discriminative Deformable Models. Machine Graphics and Vision 15(3-4), 567-576 (2006)

15. Yau, W.H., Kumar, D.K., Chinnadurai, T.: Lip-Reading Technique Using SpatioTemporal Templates and Support Vector Machines. In: Ruiz-Shulcloper, J., Kropatsch, W.G. (eds.) CIARP 2008. LNCS, vol. 5197, pp. 610-617. Springer, Heidelberg (2008)

16. Joumun, F.E., Gnanayutham, P., George, J.: Multimedia Interfaces for BSL Using Lip Readers. In: Miesenberger, K., Klaus, J., Zagler, W.L., Karshmer, A.I. (eds.) ICCHP 2008. LNCS, vol. 5105, pp. 663-669. Springer, Heidelberg (2008)

17. Kubanek, M.: Method of Speech recognition and Speaker Identification with Use Audio-Visual of Polish Speech and Hidden Markov Models. In: Saeed, K., et al. (eds.) Biometrics, Computer Security Systems and Artificial Intelligence Applications, pp. 45-55. Springer, Heidelberg (2006)

18. Choraś, M.: Human Lips Recognition. In: Kurzyñski, M., et al. (eds.) Computer Recognition Systems 2. Advances in Soft Computing, pp. 838-843. Springer, Heidelberg (2007)

19. Choraś, M.: Human Lips as Emerging Biometrics Modality. In: Campilho, A., Kamel, M.S. (eds.) ICIAR 2008. LNCS, vol. 5112, pp. 994-1003. Springer, Heidelberg (2008)

20. Choraś, M., Andrysiak, T.: Symmetry-based Salient Points Detection in Face Images. In: Rutkowski, L., Tadeusiewicz, R., Zadeh, L.A., Żurada, J.M. (eds.) ICAISC 2006. LNCS, vol. 4029, pp. 758-767. Springer, Heidelberg (2006)

21. Ross, A., Nandakumar, K., Jain, A.K.: Handbook of Multibiometrics. International Series on Biometrics. Springer, Heidelberg (2006)

22. Erzin, E., Yemez, Y., Tekalp, A.M., Ercil, A., Erdogan, H., Abut, H.: Multimodal Person Recognition for Human-Vehicle Interaction. IEEE Multimedia 13(2), 18-31 (2006) 